\title{
Surface ligand influenced free radical protection of superparamagnetic iron oxide nanoparticles (SPIONs) toward H9c2 cardiac cells
}

\begin{abstract}
There have been a number of studies which deal with either toxic or non-toxic nature of superparamagnetic iron oxide nanoparticles (SPIONs); however, there is no clear cut information about their exact behavior and the reasons for its dual action. The objective of the present study was to investigate the SPIONs having similar oxidation states, but varying surface ligands and their role in terms of protecting the iron-mediated toxic responses. The four different SPIONs includes: (i) SPIONs containing oleic acid (SPIONs-1), (ii) SPIONs without any surface ligand (SPIONs-2), (iii) SPIONs containing cysteamine ligand (SPIONs3), and (iv) SPIONs having both of oleic acid and cysteamine ligand. The particle size, surface functionality, and electronic oxidation states were confirmed by the HRTEM, FT-IR, and XPS analysis, respectively. On in vitro testing of all four SPIONs with H9c2 cardiomyocyte cell line, the SPIONs-2 without any surface ligand found to exhibit significant decrease in the viability of cells at a concentration of $200 \mathrm{gg} \mathrm{mL} \overline{1} 1$ for 16 -h exposure period. Further investigation of toxicity mechanism resulted in the fact that the SPIONs-2 involved in the formation of ROS due to the role played by the more electron deficient $\mathrm{Fe} 3+$ form of iron, there by decreased the glutathione release, increased DNA cleavage, and disrupted the mitochondrial transmembrane potential. However, the presence of unsaturation and/or thiol group (i SH) containing ligands on other SPIONs protected the cardiac cells from undergoing ROS-induced oxidative stress. Further, the results of the study confirming the importance of having unsaturated double bonds and/or ï SH group possessing ligands onto the surface of SPIONs by means of protecting the cells from the influence of electron deficient $\mathrm{Fe} 3+$ state of iron.
\end{abstract}

Keyword: Surface ligand; Free radical protection; Superparamagnetic iron oxide nanoparticles; SPIONS; H9c2; Cardiac cells 